Для цитирования: Куприянова И.Е., Гураль Е.С., Тузиков С.А. Клинические проявления непсихотических психических расстройств, основные типы психологических защит, возможности психофармако- и психотерапии у пациентов с онкопатологией. Сибирский вестник психиатрии и наркологии. 2021; 1 (110): 31-37. https://doi.org/10.26617/1810-3111-2021-1(110)-31-37

\title{
Клинические проявления непсихотических психических расстройств, основные типы психологических защит, возможности психофармако- и психотерапии у пациентов с онкопатологией
}

\section{Куприянова И.Е.' ${ }^{1}$ Гураль Е.С. ${ }^{1}$, Тузиков С.А. ${ }^{2}$}

${ }^{1}$ НИИ психического здоровья, Томский нацчональный исследовательский медицинский центр Российской академии наук

Россия, 634014, Томск, ул. Алеутская, 4

2 Онкологическая клиника НИИ онкологии, Томский наџиональный исследовательский медицинский иентр Российской академии наук

Россия, 634028, Томск, ул. Савиных, 12/1

\begin{abstract}
PEЗЮME
Актуальность. Онкологическая патология, характеризующаяся значительной динамикой прироста заболевших и высокой смертностью, является актуальной темой и насущной медицинской проблемой, в связи с чем предопределяет необходимость совершенствования комплексных программ лечения и реабилитации. Среди пациентов с онкологическими заболеваниями широко распространены психоэмоциональные нарушения, сомато- и психогенные психические расстройства. В статье показаны клинические особенности психических расстройств, наиболее часто встречающиеся психологические защиты в кризисной ситуации болезни и обсуждаются возможности терапии у пациентов с различной онкопатологией (рак легких, желудка и кишечника). Материал и методы. В исследовании приняли участие 104 пациента с диагнозом по МКБ-10 злокачественного новообразования лёгких (C34), желудка (C16) и кишечника (C18). Все пациенты были осмотрены психиатром с применением традиционного клинико-психопатологического метода. Для изучения предпочитаемых психологических защит использовалась методика Индекс жизненного стиля. Сравнительный анализ нозологической структуры пациентов с диагностированными психическими и поведенческими $(78,84 \%)$ расстройствами выявил невротические, связанные со стрессом и соматоформные $(36,54 \%)$, органические $(21,15 \%)$, аффективные $(19,23 \%)$ расстройства, в единичных случаях зарегистрировано расстройство личности $(1,92 \%)$. Примерно у пятой части $(21,15 \%)$ пациентов психические расстройства отсутствовали. В исследуемой простой случайной выборке пациентов с онкологической патологией обнаружены отклонения от нормативных показателей в использовании защитных механизмов: при раке желудка (6 [6,8]), кишечника и легких $(6[5,8])$ - отрицание; раке желудка, кишечника и легких $(8[6,10])$ - проекция; раке кишечника, легких $(7[5,8])$ и желудка $(5[5,8])$ - интеллектуализация; раке желудка, кишечника и легких $(6[4,8])$ - реактивное образование.
\end{abstract}

Ключевые слова: психоонкология, психические и поведенческие расстройства, междисциплинарный подход, психологические защитные механизмы, рак лёгких, рак желудка, рак кишечника, основные положения комбинированной терапии, комплексные программы.

\section{ВВЕДЕНИЕ}

По данным экспертов (совместная работа авторов из Франции, Швейцарии и Англии) Международного агентства по изучению рака, в мире в 2018 г. зарегистрировано 18,1 млн новых случаев онкологических заболеваний и 9,6 млн случаев смерти от рака, при этом рак лёгких, желудка и кишечника занимает лидирующие позиции по показателям заболеваемости, болезненности и смертности в ряду другой злокачественной патологии [1]. Злокачественные новообразования коморбидны с непсихотическими психическими расстройствами [2].
Злокачественные новообразования в обществе считаются смертельными, а психические расстройства «неприличными», отношение к психически больным в нашей стране предубежденностигматизирующее и даже отрицательное [3]. Негативное отношение к психическим и онкологическим заболеваниям препятствует своевременному обращению и оказанию помощи, порождает их дистанцирование и непринятие, так как в общественном сознании диагноз рака тесно связан с умиранием и вызывает целый ряд экзистенциальных проблем, связанных с переосмыслением жизни и страхом смерти. 
Снижение психического напряжения, уменьшение тревоги при травматических ситуациях в результате острой стрессовой реакции на болезнь достигается с помощью психологических защит [9]. Пациенты с онкологическими заболеваниями находятся в условиях хронического стресса, который вызван переживаниями неожиданного онкологического заболевания как такового, установлением диагноза, негативным восприятием реальности, страхами возникновения отдалённых метастазов, предстоящим изнуряющим лечением (химио- и лучевой терапией, оперативным вмешательством). Все это способствует развитию психических расстройств либо обострению существующих $[5,6,7]$.

\section{ЦЕЛЬ РАБОТЫ}

Изучение клинических проявлений психических расстройств, выявление особенностей основных типов психологических защит у пациентов с диагностированным раком лёгких, желудка и кишечника.

\section{МАТЕРИАЛЫ И МЕТОДЫ}

В исследуемую выборку были включены 104 пациента с диагностированным в соответствии с МКБ-10 злокачественным новообразованием лёгких (С34) - 24 чел., желудка (C16) - 26 чел. и кишечника (C18) - 49 чел., в возрасте от 28 и до

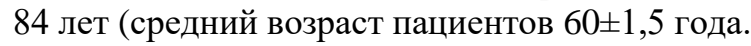

В исследование были включены пациенты, находившиеся в торакальном и абдоминальном отделениях НИИ онкологии Томского НИМЦ, готовящиеся к оперативному лечению. Простая случайная выборка носила сплошной характер. Клинический анализ психического статуса пациентов проводился с помощью общепринятого клинико-психопатологического метода. Для изучения психологических защит использовался опросник Индекс жизненного стиля (Plutchik R., Kellerman H., 1979) в интерпретации Е.Б. Клубовой [8].

\section{РЕЗУЛЬТАТЫ И ОБСУЖДЕНИЕ}

В выборке исследования большая часть приходилась на мужчин $(65-62,5 \%)$, женщин было зарегистрировано меньше (39 - 37,5\%). Онкологическая патология была представлена следующим образом: в преобладающем большинстве встречался рак кишечника (49 - 47\%), второе место принадлежало раку лёгких $(29-28 \%)$, третью позицию занимал рак желудка $(26-25 \%)$. В каждой диагностической группе (по локализации злокачественного процесса) так же характерна более высокая доля мужчин, чем женщин: с диагнозом рака кишки - 26 пациентов (53\%) и 23 пациентки (47\%), с диагнозом рака лёгких - 25 мужчин (86\%) и 4 женщины (14\%), с диагнозом рака желудка - 14 мужчин (54\%) и 12 женщин $(46 \%)$.

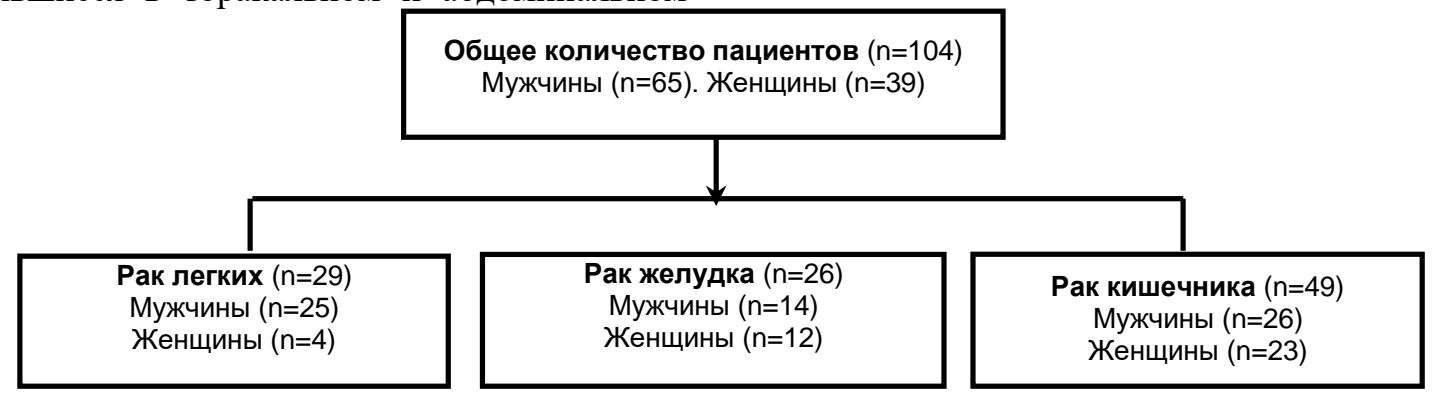

\section{Р и с у н о к 1. Распределение обследованных пациентов по локализации онкологической патологии}

Анализ количественных данных, приведенных на рисунке 1, показал, что наибольшую численность в выборке обследованных пациентов составляют больные с онкопатологией кишечника (47\%). В группе пациентов с онкопатологией легких преобладают (86\%) лица мужского пола, в двух других группах данной тенденции не наблюдается и имеет место примерно одинаковая представленность по параметру пола.

Изучение клинической картины психического состояния обследованных из выборки пациентов с диагнозом рака лёгких, желудка и кишечника показало, что среди них чаще всего встречаются невротические, связанные со стрессом и соматоформные расстройства (38 - 36,54\%), на втором месте по встречаемости находились органические расстройства $(22-21,15 \%)$, далее по распространенность следовали аффективные расстройства (20 - 19,23\%). Выявлена минимальная встречаемость расстройства личности и поведения в зрелом возрасте $(2-1,92 \%)$ Менее четверти пациентов $(22-21,15 \%)$ не имели психических расстройств, данная категория была включена в связи с тем, что выборка носила сплошной характер (табл. 1).

Таким образом, невротические расстройства с большей частотой встречаются у женщин, в то время как органические расстройства более характерны для мужчин, причем во всех группах онкопатологии. Расстройства личности в данной выборке наблюдались только у пациентов с диагностированным раком кишечника. 
Т а б л и ц а 1. Сравнительное распределение встречаемости психических расстройств у пациентов с различной онкопатологией (рак легких, желудка и кишечника) в зависимости от пола (абс., \%)

\begin{tabular}{|c|c|c|c|c|}
\hline $\begin{array}{l}\text { Без психических } \\
\text { расстройств }\end{array}$ & $\begin{array}{l}\text { Невротические } \\
\text { расстройства }\end{array}$ & $\begin{array}{l}\text { Аффективные } \\
\text { расстройства }\end{array}$ & $\begin{array}{l}\text { Органические } \\
\text { расстройства }\end{array}$ & \begin{tabular}{|c|} 
Расстройства \\
личности
\end{tabular} \\
\hline \multicolumn{5}{|c|}{ Рак легких } \\
\hline $\begin{array}{c}8-7,7 \%, \text { в т.ч. } \\
8 \text { мужчин }\end{array}$ & $\begin{array}{c}7-6,73 \%, \text { в т.ч. } \\
3 \text { мужчин и } 4 \text { женщин }\end{array}$ & $\begin{array}{c}5-4,81 \% \\
\text { все мужчины }\end{array}$ & $\begin{array}{c}9-8,65 \% \\
\text { все мужчины }\end{array}$ & - \\
\hline \multicolumn{5}{|c|}{ Рак желудка } \\
\hline \begin{tabular}{|c|}
$4-3,84 \%$, в т.ч. 3 \\
мужчин и 1 женщина
\end{tabular} & $\begin{array}{c}11-10,57 \%, \text { в т.ч. } \\
3 \text { мужчин и } 8 \text { женщин }\end{array}$ & $\begin{array}{c}8-7,7 \%, \text { в т.ч. } \\
4 \text { мужчин и } 4 \text { женщин4 }\end{array}$ & $\begin{array}{c}5-4,81 \%, \text { в т.ч. } 4 \\
\text { мужчин и } 1 \text { женщина }\end{array}$ & - \\
\hline \multicolumn{5}{|c|}{ Рак кишечника } \\
\hline \begin{tabular}{|c|}
$10-9,61 \%$, в т.ч. 7 \\
мужчин и 3 женщины
\end{tabular} & $\begin{array}{c}20-19,23 \%, \text { в т.ч. } \\
6 \text { мужчин и } 14 \text { женщин }\end{array}$ & $\begin{array}{c}7-6,73 \%,, \text { в т.ч. } \\
5 \text { мужчин и } 2 \text { женщины }\end{array}$ & \begin{tabular}{|c|}
$8-7,7 \%,$, в т.ч. 5 \\
мужчин и 3 женщины
\end{tabular} & $\begin{array}{c}2-1,92 \% \\
\text { все мужчины }\end{array}$ \\
\hline \multicolumn{5}{|c|}{ Всего } \\
\hline $\mathrm{n}=22 ; 21,15 \%$ & $\mathrm{n}=38 ; 36,54 \%$ & $\mathrm{n}=20 ; 19,23 \%$ & $\mathrm{n}=22 ; 21,15 \%$ & $\mathrm{n}=2 ; 1,92 \%$ \\
\hline
\end{tabular}

У 8 пациентов из категории лиц без психических расстройств (исключительно пациенты мужского пола) наблюдались донозологические расстройства астенического типа с преобладанием психической утомляемости. У пациентов с донозологическими расстройствами выявлялись жалобы невротического круга, слабость, утомляемость, нарушения сна. Кроме того, их беспокоили тревожные мысли о будущем, диагноз онкологического заболевания вызывал негативные эмоции в виде растерянности, беспомощности, напряженности, но они не достигали уровня какоголибо психического расстройства как на протяжении всего периода наблюдения, так и во время установления диагноза и в течение госпитализаций в НИИ онкологии. Пациентов, у которых не выявлялись психические расстройства (14 из 22 $63 \%$ ), в том числе на донозологическом уровне, объединяло двойственное восприятие ситуации госпитализации в онкологический стационар. С одной стороны, они своевременно обращаются к онкологу, обдуманно и на осознанном уровне именно в НИИ онкологии Томска, охотно выполняют протокол лечения, тщательно соблюдая прием назначенных препаратов. С другой стороны, они вытесняют действительность, не считают себя онкологическими больными: «это всё как не со мной», «я тут, а рак где-то там», «доктор сказал нужно оперироваться, только поэтому и приехал». При анализе психологических защит установлено, что у всех пациентов показатели по четырем защитам превышают нормативные значения: отрицание, проекция, интеллектуализация и реактивное образование. Анализ таблицы 2 свидетельствует о том, что у пациентов с диагнозом рака желудка, кишечника и лёгких преобладают четыре МПЗ: отрицание, проекция, интеллектуализация и реактивное образование.

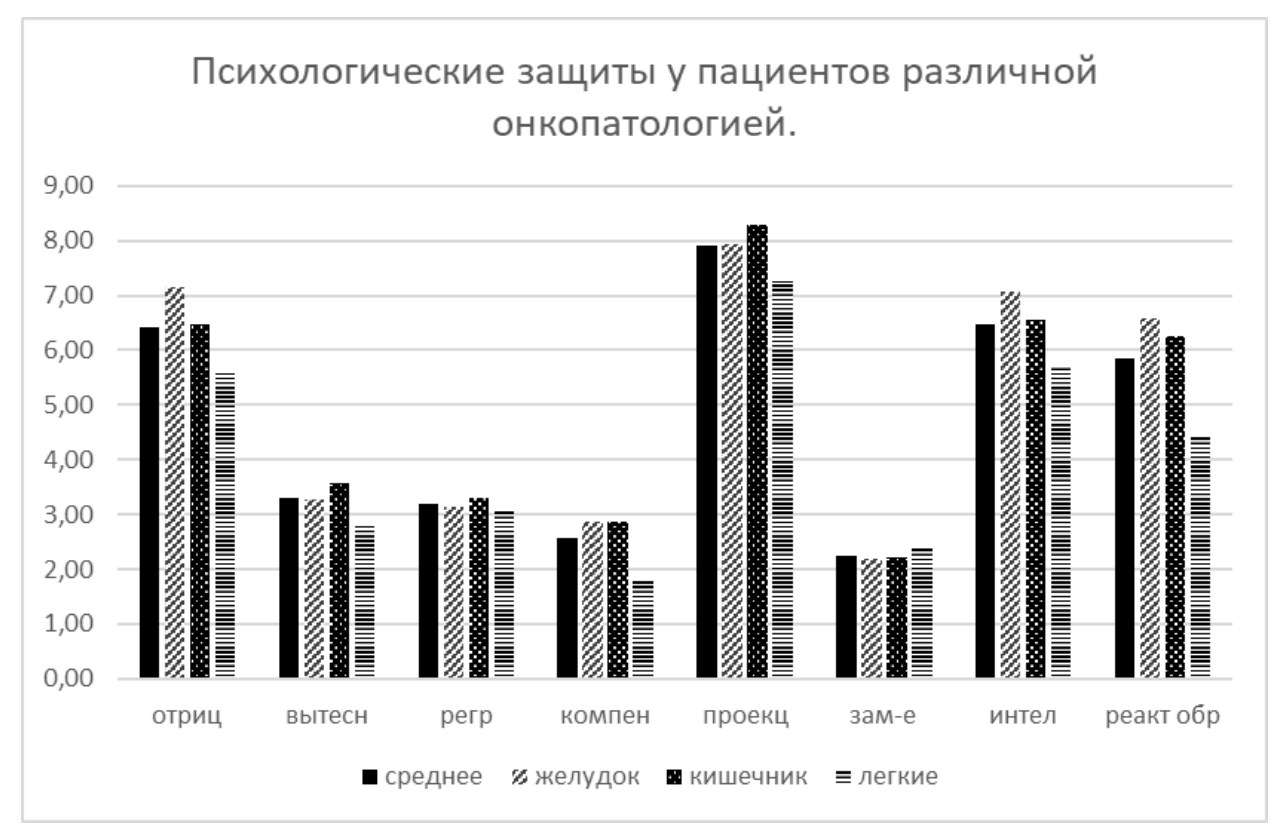


При расчётах использовали медиану (серединное ранжированное значение) в связи с неравно- мерностью групп и малым объемом выборки исследования.

T а б л и ц а 2. Сравнительное распределение встречаемости психологических защит у пациентов с различной онкопатологией (рак легких, желудка и кишечника) Медиана квартиль (25-75)

\begin{tabular}{|c|c|c|c|c|c|c|c|c|}
\hline $\begin{array}{c}\text { Вид } \\
\text { онкопатологии }\end{array}$ & Отрицание & $\begin{array}{c}\text { Вытесне- } \\
\text { ние }\end{array}$ & Регрессия & $\begin{array}{c}\text { Компенса- } \\
\text { ция }\end{array}$ & Проекция & Замещение & $\begin{array}{c}\text { Интеллек- } \\
\text { туализация }\end{array}$ & $\begin{array}{l}\text { Реактивное } \\
\text { образование }\end{array}$ \\
\hline Рак желудка & $6[6,8]$ & $3[2,5]$ & $1[1,5 ; 4]$ & $2[1,4]$ & $8[6,10]$ & $2[1,3 ; 5]$ & $5[5,8]$ & $6[4,8]$ \\
\hline Рак кишечника & $6[5,8]$ & $3[2,5]$ & $3[1,5 ; 4]$ & $2[1,4]$ & $8[6,10]$ & $2[1 ; 3,5]$ & $7[5,8]$ & $6[4,8]$ \\
\hline Рак лёгких & $6[5,8]$ & $3[2,5]$ & $3[1,5 ; 4]$ & $2[1,4]$ & $8[6,10]$ & $2[1 ; 3,5]$ & $7[5,8]$ & $6[4,8]$ \\
\hline
\end{tabular}

Высокие показатели защиты отрицание свидетельствуют о том, что пациенты бессознательно отвергают мысли о тяжести и серьёзности их состояния. С помощью проекции они, интуитивно транслируя, собственные импульсы, в том числе отрицательные, причисляют окружающим, например, соседям по палате, медицинскому персоналу, членам семьи. Благодаря реактивному образованию им удается преобразовать бессознательные импульсы во что-то другое, например, страх за свою жизнь в необходимость заботиться о своих близких, оказывать им поддержку. С помощью интеллектуализации они избавляются от мучительных и тревожных переживаний благодаря логическим построениям, чувства подменяются мыслительными процессами.

Психофармакологическая и психотерапевтическая поддержка в нашем исследовании определялась ведущим синдромом, психиатрической нозологией и проводилась с учётом полученных данных о типах психологических защит, которые так же являются психотерапевтическими мишенями. Пациенты в беседе предъявляли жалобы на сниженное настроение, нарушения сна, беспокойство о своём будущем, апатию и утомляемость. При аффективных расстройствах, в том числе органического происхождения, пациентам назначались антидепрессанты. Выбор антидепрессантов для пациентов с онкологическими заболеваниями должен учитывать совместимость психотропных препаратов с проводимой противоопухолевой терапией и их клинические эффекты седативный (миртазапин, флувоксамин, агомелатин), стимулирующий (циталопрам, флуоксетин), сбалансированный (эсциталопрам, сертралин) [4]. При невротических расстройствах психофармакологическая поддержка применялась в зависимости от тяжести психопатологической симптоматики. При выраженных симптомах тревоги и значительном снижении настроения назначались антидепрессанты, в основном из группы селективных ингибиторов обратного захвата серотонина. В случаях наличия противопоказаний выполнялась замена на антидепрессанты из других групп (например, эсциталопрам противопоказан при нарушениях свёртываемости крови и повышенной кровоточивости). При нарушениях сна и эпизодах выраженной тревоги назначался транквилизатор бензодиазепинового ряда (феназепам) в стандартной терапевтической дозировке. Пациенты с органическими расстройствами консультировались неврологом, при необходимости получали сосудистую терапию и ноотропы.

Психотерапевтическая поддержка, ориентированная на эффективные стратегии поведения, развитие уверенности в себе и веры в людей, носила индивидуальный характер. Психотерапевтический блок был разделён на несколько этапов. На первом этапе происходило знакомство с пациентом, выявление основных симптомов, жалоб, психотравмирующих ситуаций. На этом этапе решался вопрос о назначении психофармакологической поддержки и психопатологическом диагнозе. На втором этапе проходило обсуждение онкологического диагноза, выявление негативных представлений о нём. В связи с тем, что у пациентов с различной онкопатологией (рак лёгких, желудка и кишечника) выявлено практически идентичное использование одних и тех же защит (отрицание, проекция и интеллектуализация и реактивное образование), важным условием успешной работы является поиск и проговаривание переживаний, в том числе вызванных диагнозом, нахождением в стационаре, возможным изменением социального статуса (инвалидность), а также значимых психотравмирующих ситуаций, не связанных с диагнозом. Снижение напряжённости психологических защит достигается в процессе активного слушания, отражения чувств, при этом более важны приобретение новых знаний и умение интерпретировать жизненные стрессы и связанные с ними переживания, а не сами защиты [16]. Процесс принятия онкологического диагноза может занимать длительное время, однако он способствует последующей конструктивной психологической адаптации пациента, егоприверженности к лечению, принятию себя, реалистичности и ответственности за свои эмоциональные нарушения [10].

\section{ЗАКЛЮЧЕНИЕ}

По итогам исследования можно констатировать, что пациенты с психическими расстройствами, развившимися в результате онкопатологии, не склонны обращать внимание на своё пси- 
хоэмоциональное состояние, они не жалуются лечащему врачу-онкологу, не обращаются самостоятельно к специалистам в области психического здоровья.

Вероятно, это связано с высоким напряжением ведущей используемой психологической защиты отрицание. Еще одной причиной отказа от обращения за психиатрической помощью является социальная неодобряемость обществом лиц с отклонениями в состоянии психического здоровья. Более того, обращение к врачу-психотерапевту, тем более психиатру не является обыденной социальной нормой. Это можно подтвердить тем, что в нашей выборке достоверно известно только об одном пациенте, наблюдавшемся у врачапсихиатра до установления онкологического диагноза (с паническим расстройством). При этом больные погружаются в ощущение безнадёжности и мучительных переживаний, неспособности что-либо изменить в своей жизни, обращаются в бегство от реальности. Таким образом, очевидна важность практической реализации вопроса о популяризации и доступности психотерапевтической помощи для пациентов онкологического стационара (с диагнозом рака лёгких, желудка и кишечника) с тенденцией к активному преодолению трудностей и формированию жизненного оптимизма.

\section{КОНФЛИКТ ИНТЕРЕСОВ}

Авторы заявляют об отсутствии возможных конфликтов интересов в связи с публикацией материалов данной статьи.

\section{ИСТОЧНИК ФИНАНСИРОВАНИЯ}

Исследование выполнено за счет бюджетного финансирования в рамках темы «Комплексное исследование клинико-психопатологических закономерностей и патобиологических механизмов формирования и прогредиентности социально значимых психических и поведенческих расстройств с разработкой инновационных методов ранней диагностики, персонализированных стратегий терапии и профилактики» (№ AAAA-A19119020690013-2).

\section{СООТВЕТСТВИЕ ПРИНЦИПАМ ЭТИКИ}

При проведении исследования соблюдены принципы информированного согласия Хельсинской декларации ВАМ. Исследование одобрено Локальным этическим комитетом при НИИ психического здоровья Томского НИМЦ (протокол № 98 от 20 марта 2017 г.).

\section{ЛИТЕРАТУРА/REFERENCES}

1. Ferlay J, Colombet M, Soerjomataram I, Mathers C, Parkin DM, Piñeros M, Znaor A, Bray F. Estimating the global cancer incidence and mortality in 2018: GLOBOCAN sources and methods. Int J Cancer. 2019 Apr 15;144(8):1941-1953. doi: 10.1002/ijc.31937
2. Nakash O, Levav I, Aguilar-Gaxiola S, Alonso J, Andrade LH, Angermeyer MC, Bruffaerts R, Caldasde-Almeida JM, Florescu S, de Girolamo G, Gureje $\mathrm{O}$, He Y, Hu C, de Jonge P, Karam EG, KovessMasfety V, Medina-Mora ME, Moskalewicz J, Murphy S, Nakamura Y, Piazza M, Posada-Villa J, Stein DJ, Taib NI, Zarkov Z, Kessler RC, Scott KM. Comorbidity of common mental disorders with cancer and their treatment gap: findings from the World Mental Health Surveys. Psychooncology. 2014 Jan;23(1):40-51. doi: 10.1002/pon.3372

3. Шевеленкова Т.Д., Твердохлеб Н.А. Отношение к психически больным людям в современном российском обществе. Вестник Российского государственного гуманитарного университета. Серия. «Психология. Педагогика. Образование». 2015. № 2 (145). C. 139-149. Shevelenkova TD, Tverdokhleb NA. Attitude to mentally ill people in the modern Russian society. Bulletin of the Russian State Humanitarian University. Series. "Psychology. Pedagogy. Education". 2015;2(145):139-149 (in Russian).

4. Лебедева Е.В., Счастный Е.Д., Симуткин Г.Г. Комплексная реабилитация пациентов с депрессивными расстройствами, ассоциированными с онкопатологией: Медицинская технология. Томск: Издательство «Иван Фёдоров», 2016. 40 c. Lebedeva EV, Schastnyy ED, Simutkin GG. Complex rehabilitation of patients with depressive disorders associated with oncopathology: Medical technology. Tomsk: Publishing House "Ivan Fedorov", 2016: 40 (in Russian).

5. Семке В.Я., Чойнзонов Е.Л., Куприянова И.Е., Балацкая Л.Н. Развитие сибирской психоонкологии. Томск: Издательство Томского университета, 2008. 198 c. Semke VYa, Choinzonov EL, Kupriyanova IE, Balatskaya LN. Development of Siberian psycho-oncology. Tomsk: Publishing House of Tomsk University, 2008: 198 (in Russian).

6. Holland JC. Psycho-oncology: Overview, obstacles and opportunities. Psycho-Oncology, 2018 May, 27(5): 1364-1376. doi: 10.1002/pon.4692

7. Surman M, Janik ME. Stress and its molecular consequences in cancer progression. Postepy Hig Med Dosw (Online). 2017 Jun 12;71(0):485-499. doi: 10.5604/01.3001.0010.3830

8. Вассерман Л.И., Ерышев О.Ф, Клубова Е.Б., Петрова Н.Н., Беспалько И.Г., Беребин М.А., Савельева М.И., Таукенова Л.М., .Штрахова А.В., Аристова Т.А., Осадчий И.М. Психологическая диагностика индекса жизненного стиля: пособие для психологов и врачей / науч. ред. Л.И. Вассерман. СПб. : Издательский центр СПб НИ психоневрологического института им. B.М. Бехтерева. 2005. 48 с. Wasserman LI, Eryshev OF, Klubova EB, Petrova NN, Bespalko IG, Berebin MA, Savelyeva MI, Taukenova LM, Shtrakhova AV, Aristova TA, Osadchiy IM. Psychological diagnostics of the life style index: 
a guide for psychologists and doctors / scientific editor L.I. Wasserman. St. Petersburg : Publishing Center of the St. Petersburg Scientific Research Psychoneurological Institute named after V.M. Bekhterev, 2005: 48 (in Russian).

9. Прангишвили А.С., Шерозия А.Е., Бассин Ф.В. Роль неосознаваемой психической деятельности в развитии и течении соматических клинических синдромов. Бессознательное: природа, функции, методы исследования. Тбилиси: Издво «Мецниереба», 1978. Т. 2. С. 195-216. Prangishvili AS, Sherozia AE, Bassin FV. The role of unconscious mental activity in the development and course of somatic and clinical syndromes. The unconscious: nature, functions, research methods. Tbilisi: Metsniereba Publishing House, 1978;2:195216 (in Russian).

10. Заливин А.А., Набока М.В., Волосникова Е.С. Психолого-психотерапевтические аспекты реабилитации онкологических пациентов. Омский психиатрический журнал. 2019. № 3 (21). С. 3640. Zalivin AA, Naboka MV, Volosnikova E.S.
Psychological and psychotherapeutic aspects of recovery in oncological patients. Omsk Psychiatric Journal. 2019;3(21):36-40 (in Russian).

11. Чуркин А.А., Мартюшов А.Н. Практическое руководство по применению МКБ-10 в психиатрии и наркологии. М. : Изд-во МегаПро: 2010. C. 132. Churkin AA, Martyushov AN. A practical guide to the use of ICD-10 in psychiatry and narcology. Moscow: MegaPro Publishing House: 2010:132 (in Russian).

12. Мак-Вильямс Н. Психоаналитическая диагностика: Понимание структуры личности в клиническом процессе. М.: Изд-во «Класс», 2010. C. 480. McWilliams N. Psychoanalytic diagnostics: Understanding the structure of personality in the clinical process. Moscow: Publishing House "Class", 2010:480 (in Russian).

Поступила в редакцию 10.11.2020 Утверждена к печати 03.03.2021

Куприянова Ирина Евгеньевна, доктор медицинских наук, профессор, ведущий научный сотрудник отделения пограничных состояний. Author ID Scopus 6603689461/57201322290/57201322289. ORCID iD 0000-0003495-7811. Author ID РИНЦ 551544. SРIN-код РИНЦ 1617-6349.

Гураль Евгения Сергеевна, младший научный сотрудник отделения пограничных состояний. SPIN-код РИНЦ 6495-6828. Author ID 695002.

Тузиков Сергей Александрович, доктор медицинских наук, профессор, заведующий торакальным отделением. SPIN-код РИНЦ 5662-6431. Researcher ID WOS D-1176-2012. Author ID Scopus 6507842873.

Куприянова Ирина Евгеньевна, irinakupr@rambler.ru 
For citation: Kupriyanova I.E., Gural E.S., Tuzikov S.A. Clinical manifestations of non-psychotic mental disorders, the main types of psychological defenses, the possibilities of psychopharmaco- and psychotherapy in patients with oncopathology. Siberian Herald of Psychiatry and Addiction Psychiatry. 2021; 1 (110): 31-37. https://doi.org/10.26617/1810-3111-2021-1(110)-31-37

\title{
Clinical manifestations of non-psychotic mental disorders, the main types of psychological defenses, the possibilities of psychopharmaco- and psychotherapy in patients with oncopathology
}

\author{
Kupriyanova I.E.' ${ }^{1}$, Gural E.S. ${ }^{1}$, Tuzikov S.A. ${ }^{2}$ \\ ${ }^{1}$ Mental Health Research Institute, Tomsk National Research Medical Center, Russian Academy of Sciences \\ Aleutskaya Street 4, 634014, Tomsk, Russian Federation \\ ${ }^{2}$ Cancer Clinic Research Institute of Oncology, Tomsk National Research Medical Center, Russian Academy of Sciences \\ Savinykh Street 12/1, 634028, Tomsk, Russian Federation
}

\begin{abstract}
Background. Oncological pathology, characterized by the significant dynamics of increase in the number of cases and high mortality, is a relevant topic and an urgent medical problem, in connection with which it predetermines the need to improve complex programs of treatment and rehabilitation. Psychoemotional disorders, somato- and psychogenic mental disorders are widespread among patients with oncological diseases. The paper shows the clinical features of mental disorders, the most common psychological defenses in the crisis situation of the disease and discusses the possibilities of therapy in patients with various oncopathologies (cancer of the lungs, stomach and intestines). Material and Methods. The study involved 104 patients with ICD-10 diagnosis of malignant neoplasms of the lungs (C34), stomach (C16) and intestines (C18). All patients were examined by a psychiatrist using a traditional clinical psychopathological method. To study the preferred psychological defenses, the Lifestyle Index method was used. Comparative analysis of the nosological structure of patients with diagnosed mental and behavioral (78.84\%) disorders revealed neurotic, stress-related, and somatoform (36.54\%), organic (21.15\%) and affective (19.23\%) disorders. in some cases, a personality disorder was registered (1.92\%). Approximately one fifth (21.15\%) of patients had not any mental disorder. In the studied simple random sample of patients with oncological pathology, deviations from the standard indicators in the use of defense mechanisms were found: with cancer of the stomach (6 [6.8]), intestines and lungs (6 [5.8]) - denial; cancer of the stomach, intestines and lungs (8 [6.10]) - projection; cancer of the intestine, lungs (7 [5.8]) and stomach (5 [5.8]) - intellectualization; cancer of the stomach, intestines and lungs (6 [4.8]) - reactive formation.
\end{abstract}

Keywords: psycho-oncology, mental and behavioral disorders, interdisciplinary approach, psychological defense mechanisms, lung cancer, stomach cancer, bowel cancer, the main provisions of combination therapy, complex programs.

\author{
Received November 10.2020 \\ Accepted March 03.2021
}

Kupriyanova Irina E., MD, Prof, lead researcher of the Borderline State Department, Mental Health Research Institute, Tomsk National Research Medical Center, Russian Academy of Sciences, Tomsk, Russian Federation. Author ID Scopus 6603689461/57201322290/57201322289. ORCID iD 0000-0003-495-7811. RISC Author ID 551544. RISC SPIN-code 1617-6349.

Gural Evgeniya S., junior researcher of the Borderline State Department, Mental Health Research Institute, Tomsk National Research Medical Center, Russian Academy of Sciences, Tomsk, Russian Federation. RISC SPIN-code 6495-6828. RISC Author ID 695002.

Tuzikov Sergey A., MD, Prof, Head of the Thoracic Department, Cancer Clinic of Cancer Research Institute, Tomsk National Research Medical Center, Russian Academy of Sciences, Tomsk, Russian Federation. RISC SPIN-code 5662-6431. Researcher ID D-1176-2012. Scopus Author ID 6507842873.

Kupriyanova Irina E., irinakupr@rambler.ru 